\title{
CXCR4 Controls Ventral Migration of Sympathetic Precursor Cells
}

\author{
Jennifer C. Kasemeier-Kulesa, ${ }^{1,2}$ Rebecca McLennan, ${ }^{2}$ Morgan H. Romine, ${ }^{2}$ Paul M. Kulesa, ${ }^{2,3 *}$ and Frances Lefcort ${ }^{1 \star}$ \\ ${ }^{1}$ Department of Cell Biology and Neuroscience, Montana State University, Bozeman, Montana 59717, ${ }^{2}$ Stowers Institute for Medical Research, Kansas City, \\ Missouri 64110, and ${ }^{3}$ Department of Anatomy and Cell Biology, University of Kansas School of Medicine, Kansas City, Missouri 66160
}

The molecular mechanisms that sort migrating neural crest cells (NCCs) along a shared pathway into two functionally discrete structures, the dorsal root ganglia and sympathetic ganglia (SGs), are unknown. We report here that this patterning is attributable in part to differential expression of the chemokine receptor, CXCR4. We show that (1) a distinct subset of ventrally migrating NCCs express CXCR4 and this subset is destined to form the neural core of the sympathetic ganglia, and (2) the CXCR4 ligand, SDF-1, is a chemoattractant for NCCs in vivo and is expressed adjacent to the future SGs. Reduction of CXCR4 expression in NCCs disrupts their migration toward the future SGs, whereas overexpression of CXCR4 in non-SG-destined NCCs induces them to migrate aberrantly toward the SGs. These data are the first to demonstrate a major role for chemotaxis in the patterning of NCC migration and demonstrate the neural crest is composed of molecularly heterogeneous cell populations.

\section{Introduction}

The sympathetic nervous system plays a crucial role in maintaining the homeostasis of an organism. Sympathetic neurons derive from trunk neural crest cells (NCCs), a highly migratory, multipotent population of precursor cells that generate many components of the peripheral nervous system (PNS) (LeDouarin and Kalcheim, 1999). How trunk NCCs are guided through the embryo to precisely position and assemble the sympathetic ganglia (SGs) has been unclear.

After delaminating from the neural tube, NCCs navigate along two discrete migratory routes within the trunk (for review, see Kulesa et al., 2009). Medioventral migrating NCCs generate the more distally positioned SGs and the proximally positioned dorsal root ganglia (DRGs); later arising presumptive melanocytes follow a dorsolateral path (for review, see LeDouarin and Kalcheim, 1999).

NCCs destined to colonize DRGs and SGs migrate along a shared pathway, overlapping both in time and space. The cues that guide SG-destined NCCs ventrally have yet to be determined nor is it understood what makes NCCs destined for the DRGs stop more dorsally than their SG-destined cohort. Deletion of genes for neuregulin and semaphorin 3 alter the site of differentiation of sympathetic neurons (Britsch et al., 1998; Kawasaki et al., 2002), indicating separate mechanisms for guidance and differentiation, but no guidance signal has been identified that differentially sorts SG- versus DRG-destined pools of NCCs. It is

\footnotetext{
Received Feb. 17, 2010; revised July 23, 2010; accepted July 29, 2010.

This work was supported by National Science Foundation Grant I0S-0719251 (F.L.) and by the Stowers Institute for Medical Research (P.M.K.). We thank Cameron Cooper for imaging assistance.

*P.M.K. and F.L. contributed equally to this work.

Correspondence should be addressed to either of the following: Paul M. Kulesa at the above address, E-mail: pmk@stowers.org; or Frances Lefcort at the above address, E-mail: lefcort@montana.edu. DOI:10.1523/JNEUROSCI.0892-10.2010

Copyright $\odot 2010$ the authors $\quad 0270-6474 / 10 / 3013078-11 \$ 15.00 / 0$
}

critical that trunk NCCs migrate ventrally to the dorsal aorta because it is the source of local signals such as BMP4 that induce their differentiation into sympathetic neurons (Reissmann et al., 1996; Howard et al., 2000). Thus, an outstanding question is what are the molecules attracting NCCs toward the dorsal aorta?

Although inhibitory molecules in the caudal somite sculpt initial trunk NCC migratory streams through the rostral somite (for review, see Kuriyama and Mayor, 2008), it is not clear how NCCs reach distal locations. During enteric nervous system development, NCCs are attracted to the gut entrance by Shh (Reichenbach et al., 2008) and travel long distances throughout the elongating gut, mediated by netrin and GDNF (glial cellderived neurotrophic factor), which act as chemoattractants (Young et al., 2001, 2004; Jiang et al., 2003). However, whether true chemotaxis mediates NCC migration and patterning in vivo has not been determined.

Since chemokines, a superfamily of small glycoproteins with chemoattractant ability, control multiple events during embryonic development including directed cell migration (Bagri et al., 2002; Tran et al., 2004; Belmadani et al., 2005; Miller et al., 2008; Raz and Mahabaleshwar, 2009), we sought to investigate their role in patterning the PNS. We show here with gain- and loss-offunction experiments, combined with intravital time-lapse imaging, that differential expression of CXCR4 and its ligand SDF-1 sculpt a common stream of migrating NCCs into two discrete structures, the SGs and DRGs. Furthermore, we show the CXCR4 + subset of SG cells comprise the neuronal core of the SGs while later migrating SG-destined NCCs that do not express CXCR4 colonize the non-neuronal perimeter. These data provide strong evidence for chemotaxis in directing trunk NCC migration in vivo and provide the first identification of an SG target guidance cue. Last, these data provide a mechanism for regulating the balance of DRG- and SG-destined populations of NCCs. 
A

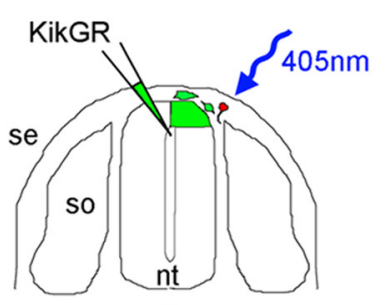

no

Ant.

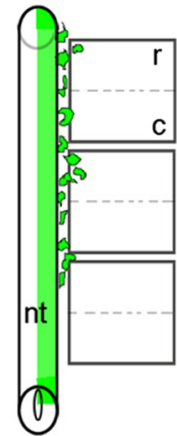

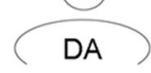

Pre-

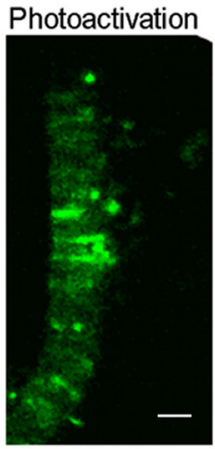

Post-
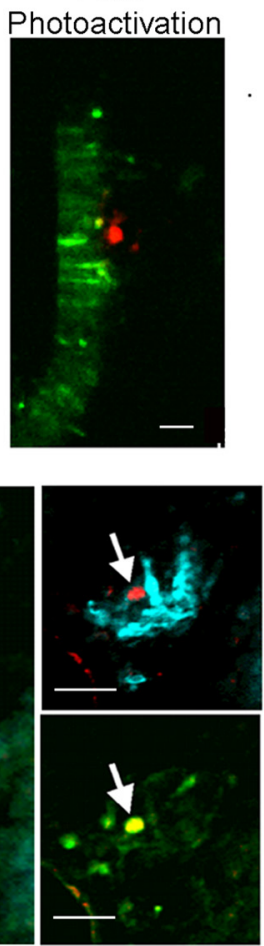

B

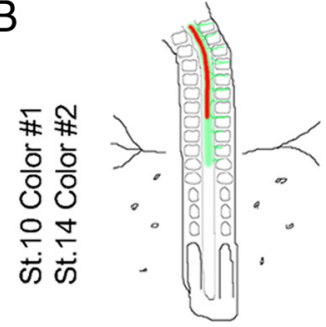

St.10 DiO St.14 Dil

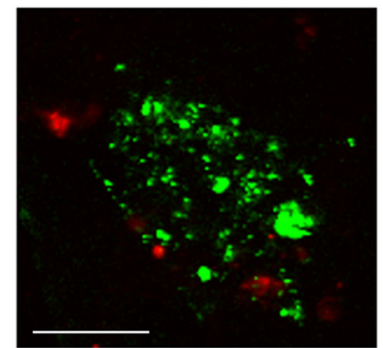

C

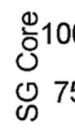

ठे 100
仓 75

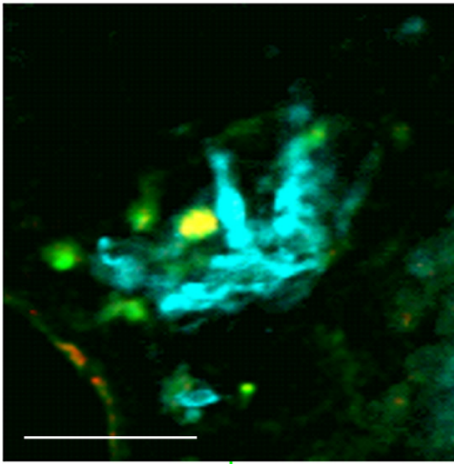

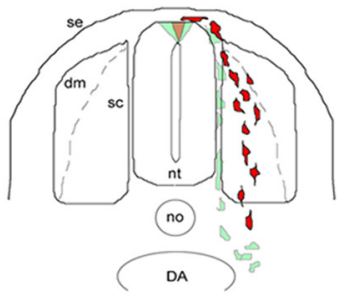

\section{St.10 EGFP St.14 RFP}
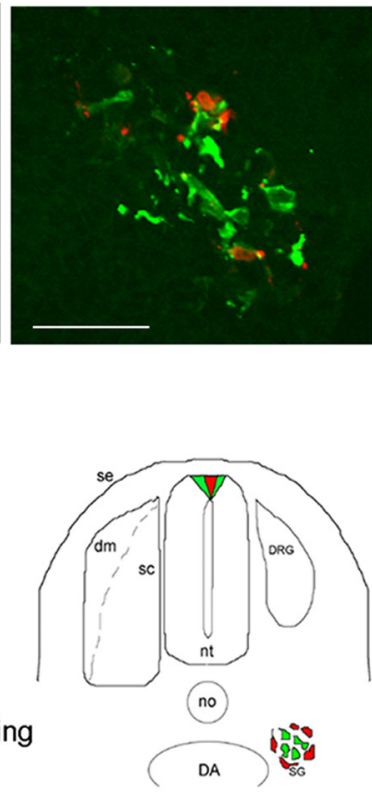

Figure 1. Early and late emigrating trunk NCCs localize to specific regions of the SGS. A, Schematic of in ovo photoactivation paradigm of KikGR, green (unphotoactivated) to red (photoactivated) conversion. Pre-photoactivation (green) image of trunk NCCs beginning to emerge from the neural tube in ovo. Post-photoactivation (red) of recently delaminated trunk neural crest cell in ovo. The bottom panel shows location of photoactivated (red) trunk NCC found in SG neural core after $48 \mathrm{~h}$ of reincubation, stained with BEN (neural; blue). B, Two-color labeling approach at HH St. 10 (color 1) and HH St. 14 (color 2) of premigratory trunk NCCS shows cells labeled at HH St. 14 localize to the perimeter of the SGs. C, Summary of cell counts of location of early versus later migrating trunk NCCs within the SGs. A percentage of $87 \pm 5.7 \%$ ( $n=10$ embryos, 18 cells counted) of early photoactivated cells were found in the neural core versus $9 \pm 6.2 \%$ ( $n=8$ embryos, 74 cells counted) of later migrating cells. Schematic to the right of the graph highlights early migrating (green) NCCs localizing to neural core, and later migrating (red) NCCs localizing to the SG perimeter. Scale bars, $50 \mu \mathrm{m}$. Error bars indicate SEM. se, Surface ectoderm; so, somite; nt, neural tube; no, notochord; DA, dorsal aorta; dm, dermamyotome; sc, sclerotome.

\section{Materials and Methods}

Embryos. Fertilized White Leghorn chicken eggs (Ozark Hatchery) were placed in a rocking incubator at $37^{\circ} \mathrm{C}(\mathrm{Kuhl})$ until appropriate developmental stages. Eggs were rinsed with $70 \%$ alcohol, and $3 \mathrm{ml}$ of albumin was removed using a $5 \mathrm{ml}$ syringe fitted with an 18 gauge needle, windowed and staged according to Hamburger and Hamilton (1951). Embryos were injected and electroporated (Stark and Kulesa, 2007) with an enhanced green fluorescent protein (EGFP)-encoding plasmid, pMES, short hairpin RNA (shRNA)-EGFP, full-length (FL)-CXCR4-EGFP, or KikGR construct to fluorescently label premigratory NCCs located in the dorsal neural tube. pMES is a control EGFP empty vector that uses the chick $\beta$-actin promoter and internal ribosome entry site, and KikGR is a photoactivatable fluorescent protein (Kulesa et al., 2008). In some experiments, a lipophilic dye, 1,1'-dioctadecyl-3,3,3',3'-tetramethylindocarbocyanine perchlorate (DiI) (incorporated into cell membranes; C-7000; Invitrogen), was mixed with plasmid DNA and coinjected into the lumen of the neural tube. For labeling of early and late premigratory NCCs with lipophylic dyes, em- bryos were injected at Hamburger and Hamilton stage 10 (HH St. 10) with $3,3^{\prime}$-dioladecyloxacarbocyanine perchlorate $(\mathrm{DiO})$ and at HH St. 14 with DiI, by microinjection, and embryos were harvested at HH St. 24. Eggs were resealed with adhesive tape and incubated at $38^{\circ} \mathrm{C}$ for $2-3 \mathrm{~d}$. After incubation, embryos were evaluated before manipulation for brightness and uniformity of EGFP label using a fluorescein isothiocyanate (FITC) filter to observe green fluorescent protein (GFP)-positive cells on a Zeiss Axiovert microscope with a $10 \times / 0.3$ (Carl Zeiss) objective, and embryos that were well labeled and developing normally were selected.

Isolation of EGFP-positive NCCs using high-purity FACS methodology. Trunk neural tubes were electroporated with control EGFP-plasmid or CXCR4 shRNA-EGFP plasmids, as described previously. After $\sim 36 \mathrm{~h}$ of reincubation, embryos were harvested, membranes removed, and trunk regions isolated by glass needle microdissection in cold Ringer's. The entire trunk region between the forelimbs and hindlimbs were excised and prepared into a single-cell suspension by treatment with $0.25 \%$ trypsin with EDTA at $37^{\circ} \mathrm{C}$ for $3 \mathrm{~min}$. This reaction was stopped by addition 


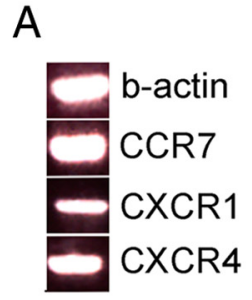

E4 SG

\begin{tabular}{l|c|c|} 
B & DRG & SG \\
\hline CCR7 & ++ & ++ \\
\hline CXCR1 & - & + \\
\hline CXCR4 & - & ++ \\
\hline
\end{tabular}

Expression symbols: ++strong, + diffuse aint, -absent

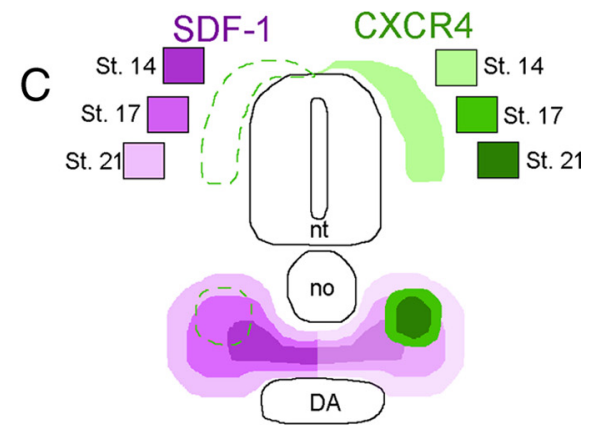

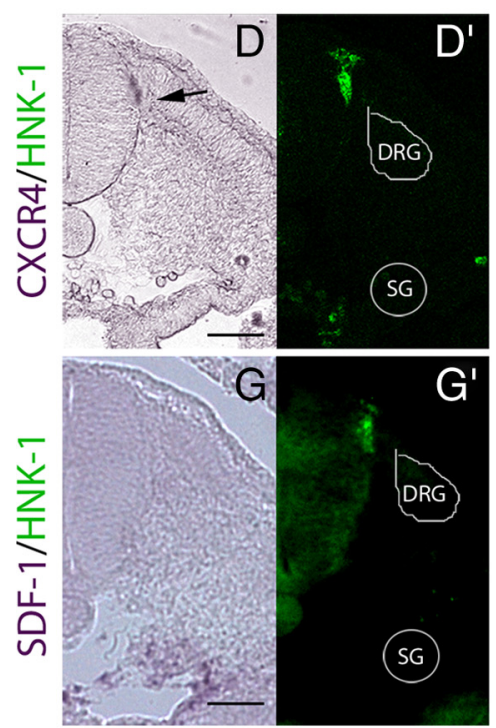

HH St.14

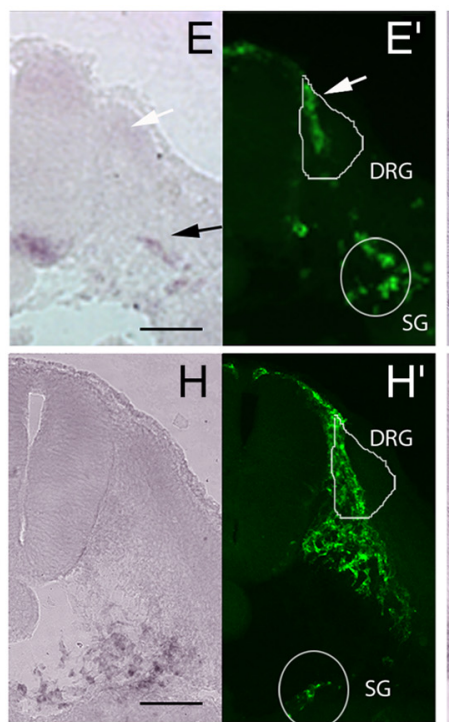

HH St. 16

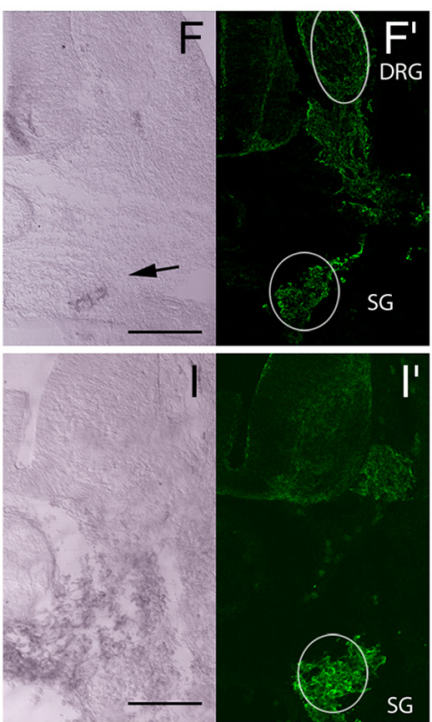

HH St. 24

Figure 2. Expression analysis of chemokine receptors during SG development. $A$, RT-PCR expression of $\beta$-actin, CCR7, CXCR1, and CXCR4 in E4 chick SGs. $B$, Table summarizing expression of CCR7, CXCR1, and CXCR4 in DRGs and SGs of E4 chick embryos. C, Schematic of transverse section through embryonic trunk region summarizing in situ expression patterns of CXCR4 (green shades) and SDF-1 (purple shades) at HH St. 14, 16, and 24. D-F, In situ hybridization of CXCR4 (purple precipitate) on chick trunk tissue at HH St. 14, 16, and 24 stained with HNK-1 on the same sections ( $\boldsymbol{D}^{\prime}-\boldsymbol{F}^{\prime}$; NCCs; green). The black arrow indicates level of NCCS and CXCR4 expression, and the white arrow indicates later migrating NCCs that do not express CXCR4. G-I, In situ hybridization of SDF-1 (purple precipitate) on chick trunk tissue at HH St. 14, 16, and 24 stained with HNK-1 ( $G^{\prime}-I^{\prime}$; green). The arrowheads indicate SGs or SG-destined NCCs. nt, Neural tube; no, notochord; DA, dorsal aorta. Scale bars, $75 \mu \mathrm{m}$.

of fetal bovine serum (FBS) (10437-010; Invitrogen) to make a 10\% final volume concentration. The cells were then centrifuged at $1000 \mathrm{rpm}$ for 5 min and resuspended in PBS with 2\% FBS. The resulting single-cell suspension was stained with $2 \mu \mathrm{g} / \mathrm{ml}$ 7AAD ( 7 -amino-actinomycin D) to label and exclude dead and dying cells, and sorted using a MoFlo cytometer (Dako). A total of 5000 EGFP-positive and EGFP-negative cells were collected from each sample.

Reverse transcription-PCR analysis. Reverse transcription (RT)-PCR was used to examine the expression of chemokine receptors and ligands in the trunk. RNA was isolated from 5775 FACS-sorted trunk NCCs. Briefly, embryos where electroporated with EGFP to label premigratory NCCs. After trunk neural crest cell migration commenced, embryos were harvested, neural tubes discarded (leaving only NCCs that had migrated into the periphery of the embryo), and FACS was used to collect only EGFP-positive cells. RT-PCR was performed using primers (Invitrogen) listed below. Resulting DNA products were $\sim 500 \mathrm{bp}$. Touchdown PCR was performed using the following conditions: $94^{\circ} \mathrm{C}$ for $5 \mathrm{~min}$ followed by 34 cycles of $94^{\circ} \mathrm{C}$ for $1 \mathrm{~min}$, annealing temperature decreasing every 2 cycles from 78 to $62^{\circ} \mathrm{C}$ for $1 \mathrm{~min}$ and $72^{\circ} \mathrm{C}$ for $1.5 \mathrm{~min}$, and then 15 cycles of $94^{\circ} \mathrm{C}$ for $1 \mathrm{~min}, 50^{\circ} \mathrm{C}$ for $1 \mathrm{~min}$, and $72^{\circ} \mathrm{C}$ for $1.5 \mathrm{~min}$. Resulting PCR products were analyzed by gel electrophoresis. Primer sequences (listed $5^{\prime}-3^{\prime}$ ) used were as follows: $\beta$-actin, forward, CGGTTTCGCCGGGGACGATG, and reverse, CGTCAGGTCACGGCCAGCCAGA; CXCR4, forward, GGACGGCCCGGACCTACTCG, and reverse, GGGGTCGCTGGCTGTTGGTG; CCR7, forward, CCCCGGTGCTCCATCATTGT, and reverse, TCTCCCGGCAGGAGGACAGC; and CXCR1, forward,
CAGAAGCAGCGCGCCATGAA, and reverse, CCATTGGGACCCCCATGCTG.

In vitro culture experiments. For in vitro cultures, glass-bottom dishes (P35G-1.5-20-C; MatTek Corporation) were coated for 30 min with 1 $\mathrm{mg} / \mathrm{ml}$ poly-L-lysine (P6282; Sigma-Aldrich), which was then removed, and the dish was allowed to dry for $30 \mathrm{~min}$. The plates were then coated with $1 \mathrm{mg} / \mathrm{ml}$ fibronectin (F1141; Sigma-Aldrich) for $30 \mathrm{~min}$ followed by 30 min of drying. During this time, heparin-acrylic beads were washed multiple times in PBS and soaked in 10-50 ng/ml SDF-1 (R\&D Systems; 460-SD-010) or left in PBS for controls. HH St. 10 whole embryos were removed from the egg and placed in Ringer's. The trunk region was excised using a sharpened tungsten needle and placed in $1 \mathrm{mg} / \mathrm{ml}$ dispase for $15 \mathrm{~min}$ on ice. After agitation with a glass pipette to help remove most of the tissue surrounding the neural tubes, the neural tubes were transferred into Neurobasal media (21103-049; Invitrogen) supplemented with B27 (17504044; Invitrogen). Any lingering non-neural tube tissue was removed and the neural tubes were washed in Neurobasal media plus B27. The neural tubes were placed onto the coated glass-bottom dishes in a minimum of media to maximize tissue adherence for $10 \mathrm{~min}$ at $37^{\circ} \mathrm{C}$. During this time, fresh $2 \mathrm{mg} / \mathrm{ml}$ collagen (354236; BD Biosciences) was prepared following the "Alternate gelling procedure" protocol outlined in the manual accompanying the collagen. Briefly, collagen was added to $10 \times \mathrm{PBS}$, ice-cold $1 \mathrm{~N} \mathrm{NaOH}$, ice-cold $\mathrm{dH}_{2} \mathrm{O}$ in a sterile chilled tube, and the contents were mixed gently and stored on ice. Beads were washed in PBS and positioned adjacent to the adhered neural tubes and $150 \mu \mathrm{l}$ of collagen was placed over the cultures. If necessary, beads were pushed back 


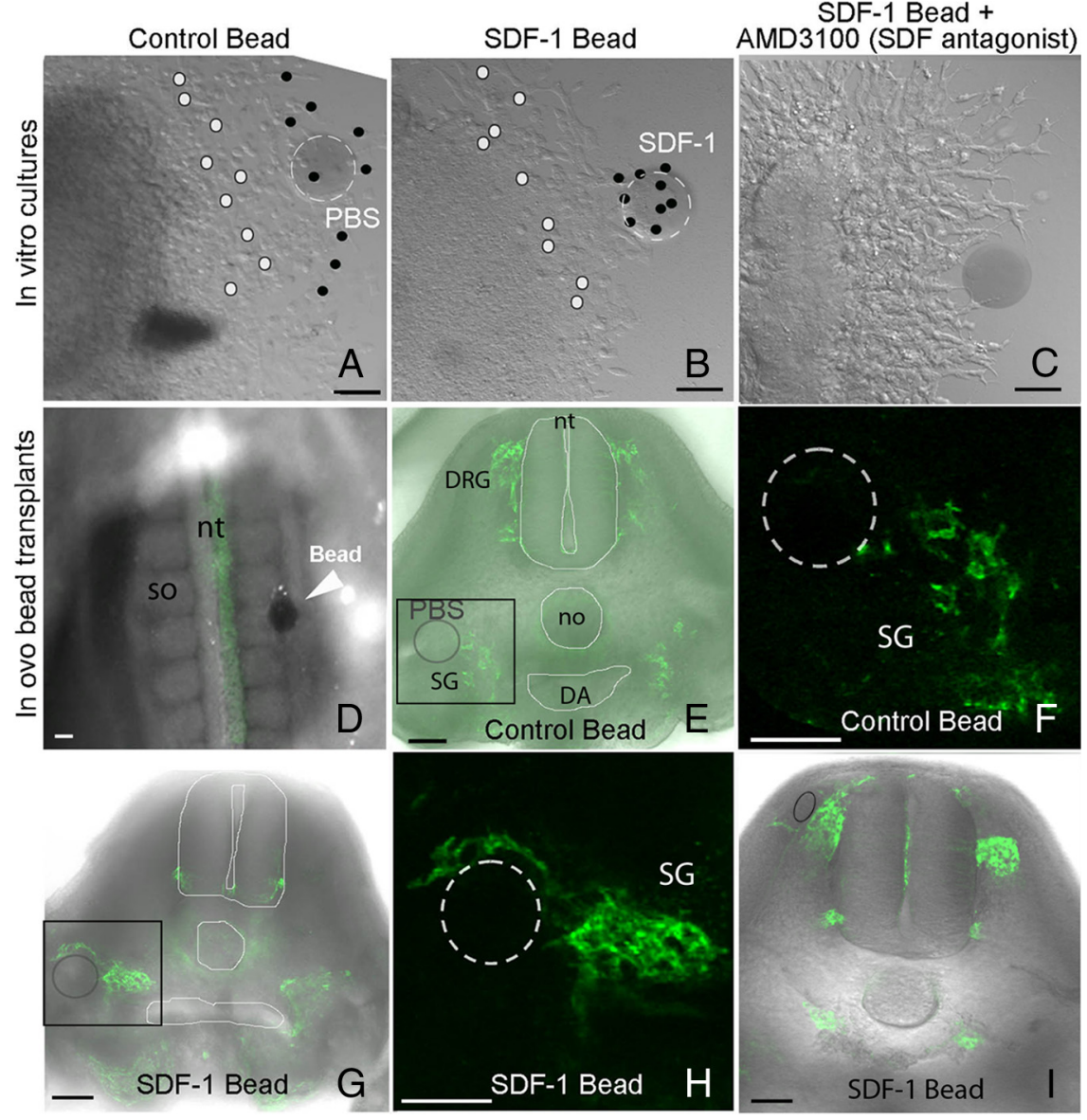

Figure 3. Attraction potential of SDF-1 on trunk NCC in vitro and in vivo. $A, B$, Time-lapse analysis of trunk neural tube cultures with control PBS- or SDF1-soaked bead adjacent to neural tube. Final image from time-lapse is shown. The white circles indicate initial position of representative population of cells, and the black circles indicate final location of cells after $16 \mathrm{~h}$. Note high density of cells surrounding SDF-1-soaked bead. C, Static image of trunk neural tube culture and SDF-1-soaked bead, in the presence of the SDF-1 antagonist, AMD3100. D- $\boldsymbol{H}$, In vivo analysis of PBS- and SDF-1-soaked beads. $\boldsymbol{D}$, Example of bead placement in vivo into HH St. 11 embryo. $\boldsymbol{E}-\boldsymbol{I}$, Images from HH St. 21 embryos. E, G, Transverse section of HH St. 21 embryo transplanted in ovo with a PBSor SDF-1-soaked bead, stained with HNK-1 (green). The black circle indicates final location of bead. $\boldsymbol{F}, \boldsymbol{H}$, Higher magnification of bead region (boxed region in $\boldsymbol{E}, \boldsymbol{G}$ ). The dotted circle indicates position of bead and arrowheads indicate ectopic NCCS. I, SDF-1 bead (black oval) in ovo placed lateral to the developing DRGs after presumptive SGs have migrated ventrally (HH St. 15). Presumptive DRG-destined neural crest cells migrate and form normal ganglia. Neural crest cells labeled with HNK-1 (green; $\boldsymbol{E}-\boldsymbol{I}$ ). Scale bars, 50 $\mu \mathrm{m}$. nt, Neural tube; no, notochord; so, somite; DA, dorsal aorta.

into place before the collagen set. The collagen was allowed to set for 10-20 min at $37^{\circ} \mathrm{C}$, and then $1.5 \mathrm{ml}$ of Neurobasal media plus B27 was added and the cultures were returned to $37^{\circ} \mathrm{C}$.

In vivo implantations. Embryos were injected and electroporated with an EGFP plasmid (pMES) at HH St. 10 and incubated for $18 \mathrm{~h}$. At this point, SDF-1 or PBS-soaked beads were implanted in ovo between the neural tube and somites, lateral to the somites, or lateral to the DRGs using fine glass pipettes and tungsten needles. After implantation, eggs were sealed and reincubated for $48 \mathrm{~h}$ (until HH St. 21), harvested, and fixed. Only embryos in which beads had stayed in place in regions where NCCs do not typically invade (such as near the notochord, mesoderm lateral to the spinal nerve and lateral to the dorsal aorta) were analyzed. Trunk regions surrounding the bead transplant site were cut into $150 \mu \mathrm{m}$ vibratome transverse sections and immunolabeled with HNK-1 to visualize all neural crest cells (see below, Immunohistochemistry).

Immunohistochemistry. Embryos were collected in PBS and fixed in 4\% paraformaldehyde for $4 \mathrm{~h}$. Embryos for cryostat sectioning were rinsed in PBS and run up in a sucrose gradient $(5,15,30 \%$ in PBS), embedded in OCT, and stored at $-80^{\circ} \mathrm{C}$. Sagittal cryostat sections were obtained at 10 $\mu \mathrm{m}$ thickness. Embryos for whole-mount staining and immunohistochemistry were fixed as above and processed as in Kasemeier-Kulesa et al. (2006). Primary antibodies included the following: HNK-1 (NCC marker), TH (tyrosine hydroxylase) (sympathetic neurons), and BEN (neuronal marker) from Developmental Studies Hybridoma Bank (University of Iowa), and Tuj1 (neuronal marker; R\&D Systems).

Probe generation. The chick pcDNA3.1CXCR4 plasmid was a kind gift from Philip M. Murphy (National Institutes of Health, Bethesda, $\mathrm{MD})$. The cCXCR4 fragment was harvested by double restriction with enzymes $\mathrm{KpnI}$ and $\mathrm{Xba}$ and inserted into pBluescript II KS vector. For the antisense probe, vector was linearized with $\mathrm{XbaI}$ and synthesized with T3 RNA polymerase. For the sense probe, vector was linearized with KpnI and synthesized with T7 RNA polymerase. The pDrive-SDF-1 probe was a kind gift from Beate Brand-Saberi (University of Freiburg, Freiburg, Germany). The antisense probe was generated by linearization with NotI and synthesized with T7 RNA polymerase, and the sense probe was linearized with KpnI and synthesized with SP6 RNA polymerase.

In situ hybridization. Embryos were fixed as above and run up in a sucrose gradient, frozen in OCT with isopentane, and cut into $25 \mu \mathrm{m}$ sections. In situ hybridization (ISH) was performed using Ventana Discovery Automated ISH System using a RiboMap kit (Ventana). Briefly, probes were incubated on slides overnight (CXCR4 used at $620 \mathrm{ng} / \mu \mathrm{l}$; SDF-1, $240 \mathrm{ng} / \mu \mathrm{l})$. Antidigoxigenin-AP fragments (Roche), 1:5000, was applied to each slide for $2 \mathrm{~h}$ and washed. The following day, slides were rinsed in $20 \mathrm{ml}$ of warm double-distilled (DD) $\mathrm{H}_{2} \mathrm{O}$ with $0.5 \mathrm{ml}$ of dish soap (Dawn; Procter \& Gamble) and washed twice in $\mathrm{DDH}_{2} \mathrm{O}$. Slides were put into NBT/BCIP (nitroblue tetrazolium/5-bromo-4-chloro-3-indolyl-phosphate) (11681451001; Roche; both at $50 \mathrm{mg} / \mathrm{ml}$ ) for 2-4 $\mathrm{h}$ for color reaction to develop.

shRNA and chick full-length CXCR4 construction. Target sequences for shRNA knockdown were designed using Ambion's Target Finder, which uses design guidelines first described by Tuschl and colleagues (Elbashir et al., 2001). The target sequences and oligonucleotides for insertion into pSilencer (listed $5^{\prime}$ to $3^{\prime}$ ) were as follows: CXCR4 target sequence, GTCGATTCTTCGAGAGCAA, and corresponding oligonucleotides, top, GTCGATTCTTCGAGAGCAATTCAAGAGATTGCTCTCGAAGAATCGA CTTTTTT, and bottom, AATTAAAAAGTCGATTCTTCGAGAGCA ATCTCTTGAATTGCTCTCGAAGAATCGACTTTTTT; and control scrambled target sequence, GATCTGACTTCTAAGGCAG, and corresponding oligonucleotides, top, GATCTGACTTCTAAGGCAGTTCAA GAGACTGCCTTAGAAGTCAGATCAATTTTTT, and bottom, AATTAAA AAATGATCTGACTTCTAAGGAGTCTCTTGAACTGCCTTAGAAGTCA GATCGGCC. A second CXCR4 shRNA construct targeted to a different region was generated to confirm results with the target sequence, GATTGGCTCAGCTGACTAT, and corresponding oligonucleotides, top, GATTGGCTCAGCTGACTATTTCAAGAGAATAGTCAGCTGAGCCAAATCT C TTTTT, and bottom, AATTAAAAAAGAGATTGGCTCAGCTGACTA TTCTCTT GAAATAGTCAGCTGAGCCAATCGGCC. After positive clones were confirmed via sequencing, the mouse U6 promoter and shRNA regions were excised and cloned into the EGFP control vector, pMES, for dual expression of the shRNA and EGFP. The chick pcDNA3.1-CXCR4 plasmid (Philip M. Murphy, National Institutes of Health, Bethesda, MD) was subcloned into pMES-EGFP, which encodes a cytoplasmic fluorescent marker. 
A

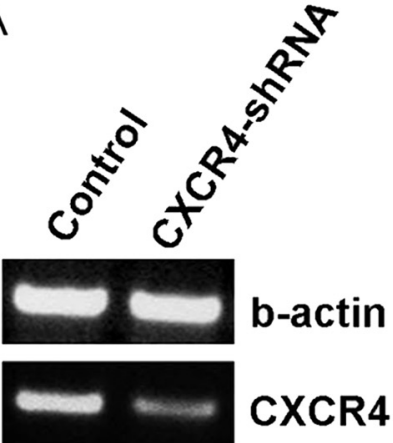

E2.5 neural crest cells
B

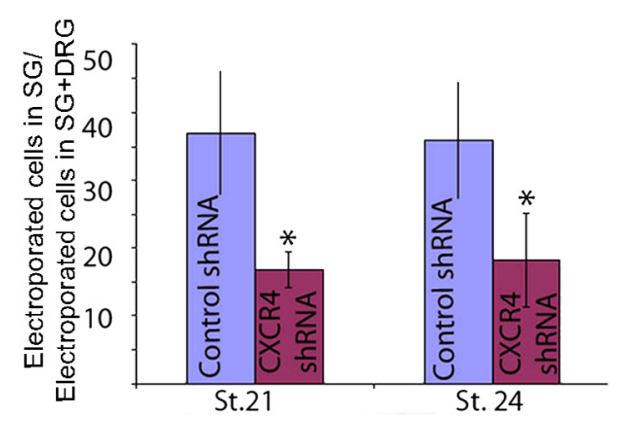

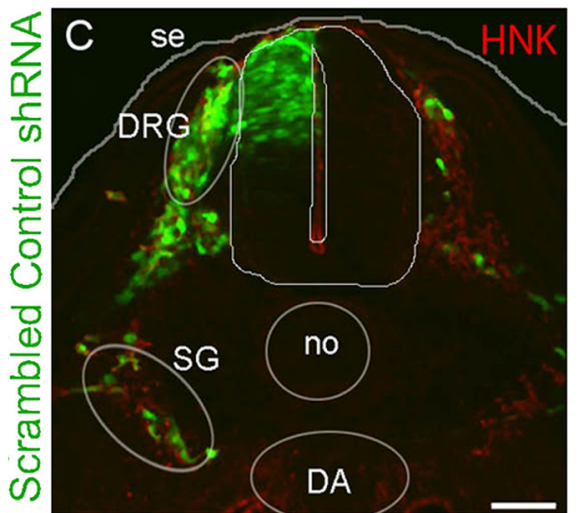

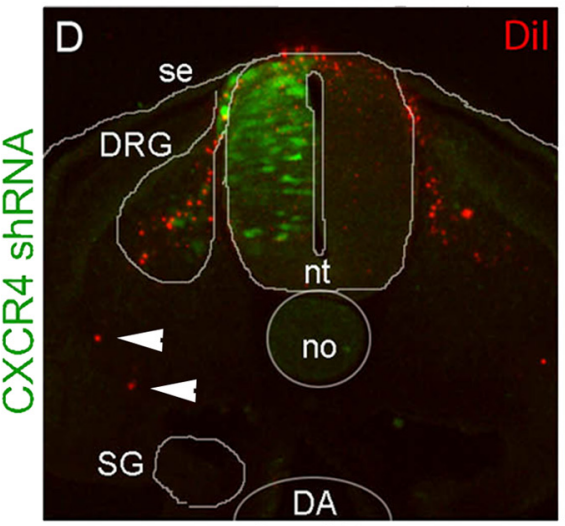

St. 15

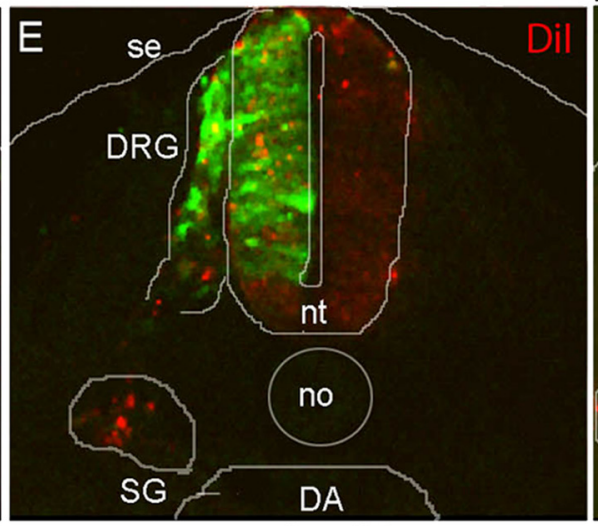

St. 17

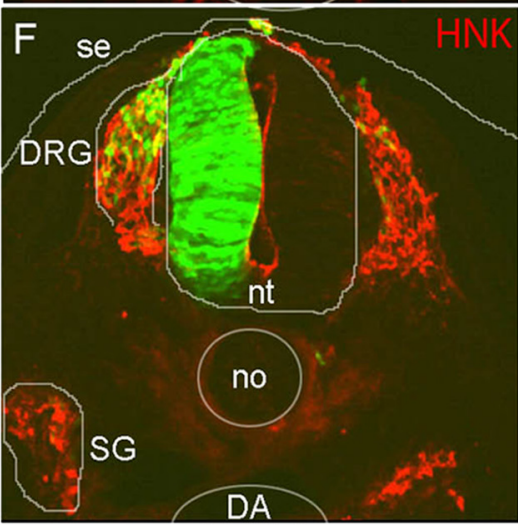

St. 21

Figure 4. Reduction in CXCR4 mRNA impairs ventral migration of trunk neural crest cells. A, RT-PCR of control $\beta$-actin and CXCR4 on FACS-sorted NCCs expressing control EGFP or CXCR4-shRNA plasmid at HH St. 16 (E2.5). B-F, Premigratory NCCs were electroporated with CXCR4-shRNA alone or with Dil. B, Percentage of electroporated NCCS (control scrambled-shRNA and CXCR4-shRNA) found in the SGs compared with the DRGs at HH St. 21 and 24. Error bars indicate SEM. C, Transverse section of HH St. 21 embryo transfected with control-shRNA plasmid at HH St. 10 shows NCCs (HNK-1; red) distributed between and within DRGs and SGS. D, E, Images of embryos coinjected with CXCR4-shRNA (green) and Dil (red) at HH St. 15 and 17. The arrowheads in $\boldsymbol{D}$ indicate Dil + /CXCR4-shRNA - cells that have migrated further ventrally than CXCR4-shRNA + cells. $\boldsymbol{F}$, Image of embryo injected with CXCR4-shRNA (green) and stained for HNK-1 (red) at HH St. 21. no, Notochord; DA, dorsal aorta; se, surface ectoderm; nt, neural tube. Scale bar: $C-F, 50 \mu \mathrm{m}$.

Three-dimensional confocal and time-lapse imaging. Three-dimensional image $z$-stacks were collected on an inverted laser-scanning confocal microscope (LSM5 Pascal; Zeiss) using either a Plan-Neofluar 10×/0.3 (Zeiss), Plan-Neofluar $40 \times / 0.75$ (Zeiss) or C-Apochromat $40 \times / 1.2 \mathrm{~W}$ objective (Zeiss). Images were visualized using AIM software (Zeiss).

For in vitro time-lapse confocal imaging, cultures were chosen based on quality of tissue, level of construct expression, and distance from bead. The top cover of the glass-bottom dish was replaced with a top containing a coverslip insert. An LSM5 Pascal (Zeiss) was used to collect single-plane images every 4 min.

For embryo explant time-lapse microscopy, the microscope was surrounded with a snug-fitting cardboard box and thermal insulation (Reflectix; BP24025) with a tabletop incubator (Lyon Electric; 950-107) fed into one side of the box (Kulesa and Kasemeier-Kulesa, 2007). The EGFP plasmid was excited with the $488 \mathrm{~nm}$ laser line using the FITC filter and all other imaging parameters were as described by Kasemeier et al. (2004).

Photoactivation experiments were performed as in the study by Kulesa et al. (2008). Briefly, embryos were injected at HH St.10 with a plasmid encoding KikGR, which undergoes a green to red fluorescence conversion when photoactivated with $405 \mathrm{~nm}$ laser excitation, and allowed to develop to desired stage. All photoconversions were performed in ovo using an upright confocal microscope (LSM 5 Pascal; Carl Zeiss). KikGRexpressing cells were located using normal $488 \mathrm{~nm}$ laser excitation and selected cells photoconverted. Eggs were then reincubated until the appropriate developmental stage.

Data analysis. Images were collected, processed, and analyzed using Zeiss AIM software. Measurement calculations were performed using AIM software. Statistical analysis was performed using Student's $t$ test. To standardize multiple datasets (because of varying sizes of DRGs) when measuring the fluorescence intensity signals, we used a grid consisting of 20 horizontal bars, and their height altered so for each DRG we only measured 20 horizontal bars (see Fig. 6). The upper limit of the grid was positioned at the dorsal root entry zone (DREZ) of each DRG analyzed, and the lower limit just above the motor exit point (ventral region of neural tube where motor axons exit and join with the peripherally growing DRG axons). We then averaged the fluorescence intensity across multiple datasets for each of the 20 horizontal bars. The distance along the dorsolateral migratory pathway was measured from the midline of the neural tube to the furthest GFP+ cell located on this migratory route. To determine the location of all transfected NCCs in both control- or CXCR4-shRNA-expressing embryos, we counted all transfected NCCs at HH St. 24 [embryonic day 4 (E4)] in the DRGs, SGs, ventral root and dorsolateral pathway on the electroporated (ipsilateral) side of the embryo and in the DRGs, SGs, and dorsolateral pathway on the nonelectroporated (contralateral) side of the embryo, and also those found in ectopic locations (where NCCs are not usually found). We calculated the number of GFP + cells residing in the dorsal root region compared with the entire DRGs by measuring the length of the DRGs (from the DREZ to the motor exit point-ventral root region). We counted those cells within the top one-fourth of the ganglia and compared this to the total number of GFP + cells in the entire DRGs.

\section{Results}

Delamination order from the neural tube determines position and cell fate within SGs

Previous studies have established a temporal order in the exodus of NCCs from the neural tube with the earliest delaminating NCCs migrating furthest ventrally to form SGs, and the last 


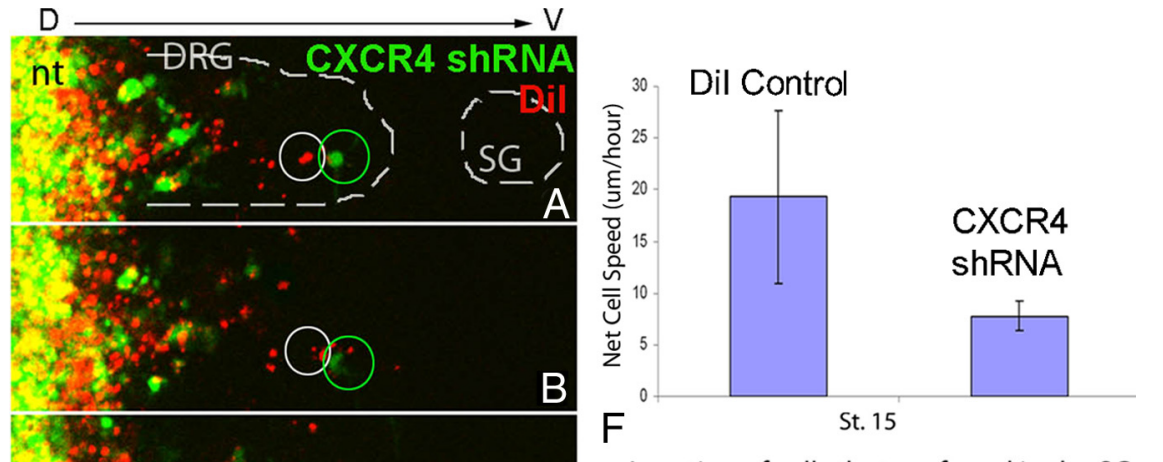

Location of cells that are found in the SG

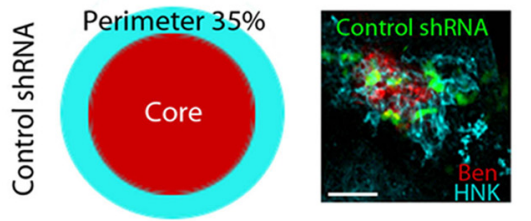

$\mathrm{D}$

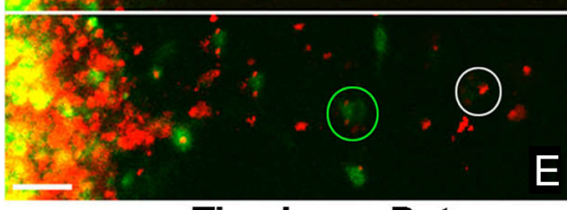

Timelapse Data

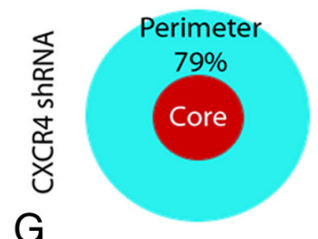

Figure 5. Role of $X X C R 4$ in NCC migration and position within the SGS. A-E, Static images from time-lapse of HH St. 13 embryo coinjected with CXCR4-shRNA (green) and Dil (red) imaged in whole-embryo explant. The white and green circles track individual Dil- and CXCR4-shRNA-labeled cell throughout time-lapse, respectively. $F$, Average cell speeds of Dil-control ( $22.1 \pm 7.6 \mu \mathrm{m} / \mathrm{h})$ and CXCR4-shRNA ( $9.8 \pm 1.5 \mu \mathrm{m} / \mathrm{h}$ )-transfected trunk NCCs from time-lapse analysis ( $n=3$ sessions). Error bars indicate SEM. G, Graphical representation of localization of control-shRNA and CXCR4-shRNA + NCCs, core (red) or perimeter (light blue), within the SGs. Fluorescent images represent typical SGs expressing control-shRNA or CXCR4-shRNA plasmids (green), labeled with BEN (red, core) and HNK-1 (blue). Scale bar, $25 \mu \mathrm{m}$. nt, Neural tube; D, dorsal; V, ventral.

NCCs to delaminate migrating dorsolaterally to form melanocytes (for review, see Serbedzija et al., 1989; LeDouarin and Kalcheim, 1999). We hypothesized that temporal differences could also pattern the relative distribution and contribution of neural and progenitor cell populations in the immature SGs. From previous work in our laboratories, we identified a novel late subpopulation of NCCs that migrates through the rostral somite and directly targets the region between two incipient SGs, before coalescing with one of the two SGs (Kasemeier-Kulesa et al., 2006). To further investigate the fate of these temporally distinct migratory waves, we differentially labeled early and late delaminating NCCs and determined their identity within the SGs.

To specifically mark the earliest trunk NCCs exiting the neural tube, we used in ovo photoactivation, labeling premigratory NCCs with KikGR (Fig. 1 A) (Stark and Kulesa, 2007). Single and small numbers of early delaminating NCCs at HH St. 13 were photoactivated in ovo (green to red fluorescence change after exposure to $405 \mathrm{~nm}$ laser excitation; 23) (Fig. 1A). After SG formation was complete ( $\sim 48 \mathrm{~h}$ after photoactivation), SGs were analyzed using neural markers. Fourteen of 18 early photoactivated cells were found in the neural core (immunopositive for neural markers) of the SGs ( $n=10$ embryos, 18 cells) (Fig. $1 C$ ), indicating the earliest delaminating NCCs (HH St. 13) colonize the core of the SG anlagen and differentiate into neurons.

To determine the fate of later delaminating NCCs that colonize the SGs, we used a two-color labeling approach (Fig. $1 \mathrm{~B}$ ). Premigratory NCCs were labeled at HH St. 10 with one fluores-

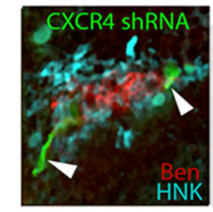

cent marker (DiO or EGFP) and at St. 14 with a second fluorescent marker [DiI or RFP (red fluorescent protein)]. After primary SG formation, most cells labeled at St. 14 (later migrating NCCs) were found along the perimeter of the SGs (Fig. 1C), with only $9 \pm 6.2 \%$ of cells found in the core ( $n=8$ embryos, 74 cells). Together, these data demonstrate that early and late delaminating NCCs destined for the SGs colonize distinct regions of the SGs with the earliest emerging NCCs forming the neuronal core and later emerging NCCs colonizing the SG perimeter.

\section{CXCR4 is expressed by a subset of trunk NCCs}

These data indicated that temporal differences in NCC delamination correlated with distinct fate within the SGs. To determine whether NCC migration patterning is also regulated by heterogeneous gene expression, we examined expression of multiple chemokine receptors in trunk NCCs. We hypothesized that, if a given chemokine receptor functioned to selectively direct the migration and targeting of SG-destined NCCs, it should not be expressed by DRGs or DRG-destined NCCs. Using RT-PCR, we found expression of CXCR4, CCR7, and CXCR1 (Fig. 2A) in $\mathrm{HH}$ St. 24 SGs. Whole-mount ISH of HH St. 24 embryos showed CCR7 highly expressed in DRGs and SGs, and CXCR1 diffusely expressed in SGs and faintly in DRGs (Fig. 2 B; supplemental Fig. 1, available at www.jneurosci.org as supplemental material). CXCR4, however, was selectively expressed in SGs and not DRGs (Fig. 2 B; supplemental Fig. $1 C$, available at www.jneurosci.org as supplemental material). Since ventrally migrating trunk NCCs typically follow a medioventral migratory route, stopping at the DRGs or SGs, selective expression of CXCR4 by NCCs that generate SGs suggested a potential novel role for chemokines in imparting a pattern on a migratory stream of NCCs.

To determine the spatiotemporal expression of CXCR4 and its ligand SDF-1 during SG development, we combined in situ hybridization with HNK-1 immunostaining at HH St. 14 (early trunk NCC migration), St. 16 (arrival of NCCs at SG sites), and St. 24 (primary SGs formed). At HH St. 14, early emerging trunk NCCs expressed CXCR4 (Fig. 2D), and SDF-1 was expressed from the dorsal aorta dorsally around the notochord just past the level of the ventral neural tube (Fig. $2 G$ ), along the migratory pathway of SG-destined NCCs. By HH St. 16, CXCR4+ NCCs localized to the presumptive SGs (cells dispersed, but adjacent to the dorsal aorta) (Fig. 2E) but more recently delaminated NCCs lacked CXCR4 expression. SDF-1 localized to the perinotochordal region (between the level of the dorsal aorta and notochord) (Fig. 2H). By HH St. 24, CXCR4+ cells were localized primarily in the neural core of the SGs (Tsarovina et al., 2008) (Fig. 2 F). Importantly, although at HH St. 24 numerous NCCs were distributed among different target locations, double labeling with HNK-1 clearly showed only a discrete subset of NCCs express CXCR4. It is not expressed in the DRGs, nor by NCCs 


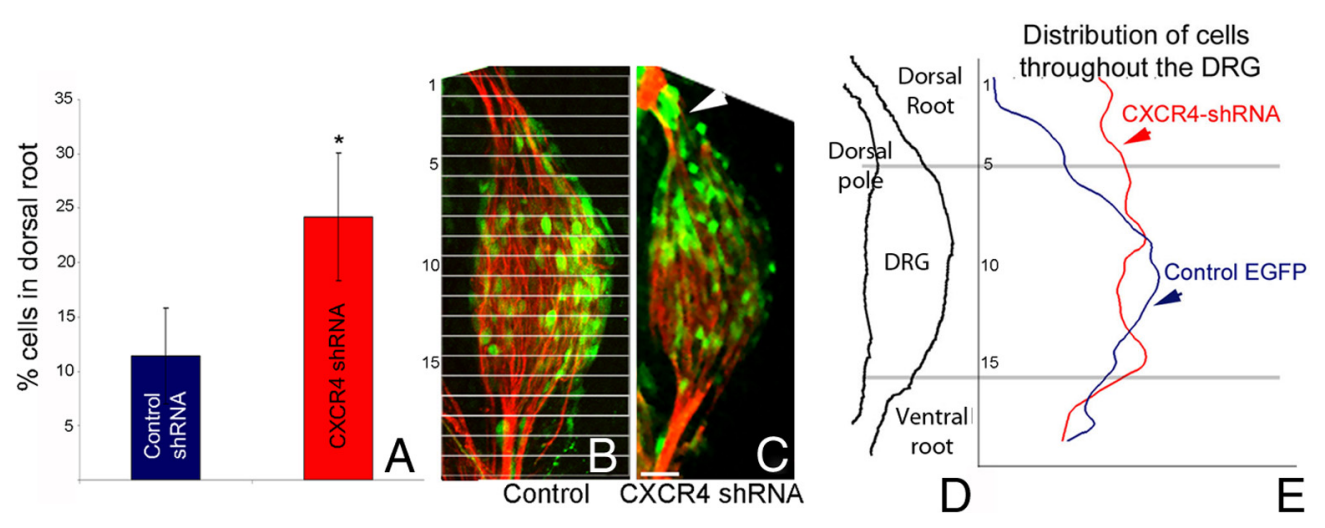

Figure 6. CXCR4-shRNA + cells accumulate in the dorsal root. A, Percentage of control and CXCR4-shRNA-expressing cells in the dorsal root, compared with entire DRGs. Length from the dorsal and ventral root was divided into four sections vertically. Cells were counted within top one-quarter section and compared with total number of cells found in all four sections. A percentage of $11.4 \pm 4.4 \%$ of control cells ( $n=4$ embryos, 541 cells) was found in the dorsal one-quarter of DRGs, versus $24.2 \pm 5.9 \%$ in CXCR4-shRNA-treated embryos ( $n=4$ embryos, 452 cells; $p \leq 0.001)$. Error bars indicate SEM. Asterisk (*) indicates significant differences $(p<0.01)$. B, C, Transverse sections of E4 embryos transfected with control- or CXCR4-shRNA (green), stained with Tuj1 (red). The arrow indicates cells localized to DREZ. D, Schematic of main regions of the DRGs; the horizontal gray lines indicate level of dorsal root. $E$, Average relative fluorescence intensity ( $x$-axis) graph through entire length of the DRGs ( $y$-axis) for control-and CXCR4-shRNA-expressing embryos. A horizontal grid was overlaid on each DRG image, and average intensity was calculated for each horizontal grid, averaged across multiple DRGs, and mapped onto a graph. The blue line represents control-shRNA fluorescence distribution ( $n=7$ embryos), and the red line indicates CXCR4-shRNA fluorescence distribution ( $n=7$ embryos). Scale bar, $25 \mu \mathrm{m}$.

comprising the SG perimeter. SDF-1 expression remained strongly localized around the notochord, tapering off laterally near the spinal nerve (Fig. 2I). These expression patterns are summarized in Figure $2 C$ and are supported by other recent in situ hybridization analyses in chick (Yusuf et al., 2005) showing strong expression of CXCR4 in developing SGs but absence of expression in immature DRGs. Based on these data, we hypothesized SDF-1 expression may guide SG precursors toward the dorsal aorta.

\section{SDF-1 is a chemoattractant for trunk NCCs in ovo}

To assess the chemoattractant activity of SDF-1 on trunk NCCs, HH St. 10 trunk neural tube explants were cultured with beads coated in PBS (control; $n=11$ ) or recombinant SDF-1 protein $(n=16)$ (Fig. 3). Using time-lapse microscopy, in the presence of a PBS-coated bead, NCCs emerged and migrated away from the neural tube in a uniformly radial direction with no apparent localization pattern with respect to the bead (Fig. $3 A$ ). However, in the presence of an SDF-1-coated bead, cells were found congregated in an asymmetric distribution around the bead (Fig. $3 B)$. To confirm the directed response to SDF-1, we also cultured neural tubes and SDF-1-coated beads with an SDF-1 antagonist 1,1' -[1,4-phenylenebis(methylene)] bis [1,4,8,11-tetra-azacyclotetradecane] octohydrobromide dihydrate (AMD3100), also an agonist for CCR7; $n=6$ ] (Kalatskaya et al., 2009), which generated a similar cell distribution pattern to controls (Fig. 3C).

To determine whether SDF-1 could act as a chemoattractant in ovo, SDF-1- or PBS-coated beads were transplanted $(n=9$ embryos, 1 bead/embryo) into the lateral somite of HH St. 11 embryos (Fig. 3D, arrowhead). At HH St. 21, NCCs populated SGs in both conditions (Fig. $3 E-H$ ). However, when beads were implanted into regions from which NCCs are typically inhibited, NCCs were only found in these ectopic locations in association with SDF-1- and not PBS-coated beads. Additionally, we tested the effect of SDF-1 on DRG-destined NCCs by transplanting SDF-1-coated beads in ovo, underneath the ectoderm near the developing DRGs at HH St. 15 after the majority of SG-destined NCCs had migrated ventrally. At HH St. 21, DRG and SG formation was unaltered, indicating DRG-destined NCCs are not influenced by SDF-1 (Fig. 3I).

\section{CXCR4 expression is required for ventral migration of SG precursors}

To disrupt CXCR4 signaling in trunk NCCs, we generated an EGFP-tagged CXCR4-shRNA construct (CXCR4-shRNA) and a plasmid encoding EGFP-tagged control-shRNA (scrambled target sequence). Embryos were electroporated in ovo with the shRNA constructs at St. 10. Confirmation of reduction in CXCR4 in neural crest cells was obtained by RT-PCR (Fig. 4A). Scrambled-shRNA transfected NCCs distributed normally throughout the DRGs and SGs ( $n=4$ embryos, 229 cells) (Fig. $4 C)$. In contrast, CXCR4-shRNA + NCCs were absent in SGs ( $n=6$ embryos, 255 cells) (Fig. $4 D-F$ ). Static analysis of CXCR4shRNA transfected embryos coinjected with DiI ( $n=4$ embryos) showed normal NCC delamination and emigration from the dorsal neural tube and migration within rostral somite halves $(\mathrm{HH}$ St. 15). However, DiI +/shRNA - cells migrated further ventrally than CXCR-shRNA+ cells (Fig. 4D).

Strikingly, very few CXCR4-shRNA + NCCs were found in the SGs compared with control-scrambled shRNA+ cells $(\mathrm{HH}$ St. 17,21$)$ and those that did were primarily located along the perimeter of the ganglion (Fig. 5G; control data for St. 17 not shown). We also measured a decrease in the distribution of NCCs between the SGs and DRGs in CXCR4-shRNA-treated embryos compared with control embryos by determining the percentage of GFP + cells that resided in the SGs compared with the total number of GFP + cells in the DRGs and SGs combined at HH St. 21 and 24 (Fig. $4 B$ ). To determine the overall migration pattern of transfected NCCs in control or CXCR4-shRNA embryos, we counted all transfected NCCs at HH St. 24 in the DRGs, SGs, ventral root, and dorsolateral pathway on the electroporated (ipsilateral) side of the embryo and in the DRGs, SGs, and dorsolateral pathway on the nonelectroporated (contralateral) side of the embryo, in addition to those found in ectopic locations. A higher percentage of CXCR4-shRNA+ cells settled within the DRGs than did control-transfected NCCs $(72.7 \pm 7.1$ vs $60.1 \pm 4.8 \%$ in controls; $p \leq 0.002)$. Conversely, we found a significant decrease in cells in the SGs compared with controls $(4.7 \pm 5.6$ vs $17.1 \pm$ $6.8 \% ; p \leq 0.001)$. However, there was no significant difference in the percentage of NCCs found in the other locations analyzed 

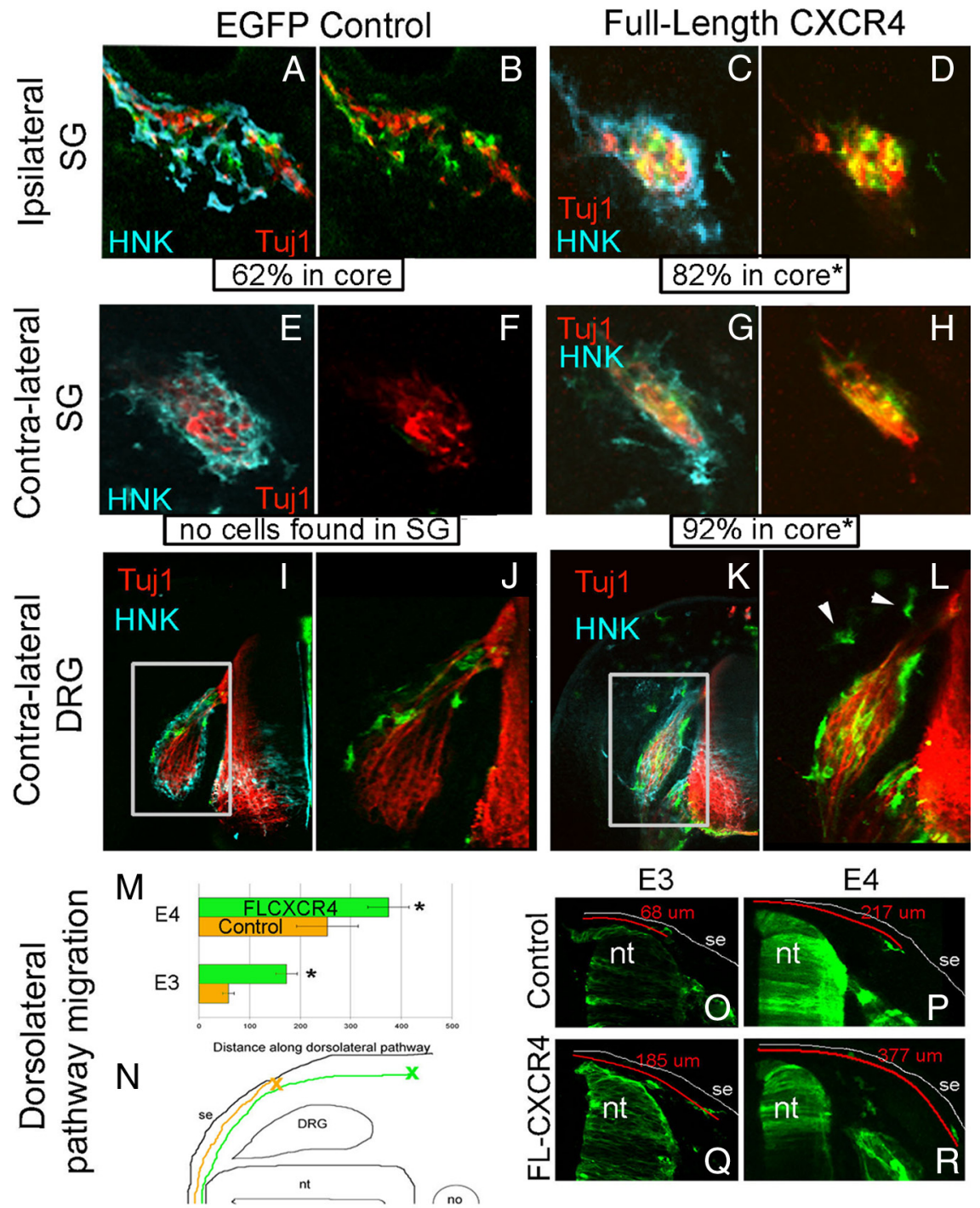

E3

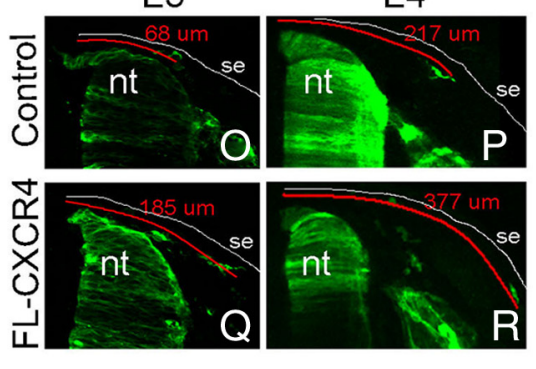

Figure 7. Overexpression of CXCR4 alters migration and localization of trunk NCCs. A-D, Analysis of FL-CXCR4 (green) on ipsilateral SG (electroporated side) formation, HNK-1 (blue) and Tuj1 (red). $\boldsymbol{E}-\boldsymbol{H}$, Analysis of contralateral SGs (un-electroporated side) in control-EGFP $(\boldsymbol{E}, \boldsymbol{F})$ and FL-CXCR4 $(\boldsymbol{G}, \boldsymbol{H})$ electroporated embryos. I-L, Analysis of contralateral DRGs of control-EGFP and FL-CXCR4 electroporated embryos. The arrowheads indicate cells found in ectopic locations lateral to the DRGs. M-R, FL-CXCR4+ NCCs migrate further along the dorsolateral pathway than control-EGFP + NCCS. At E3, control-EGFP + NCCs are found $58.5 \pm$ $11.1 \mu \mathrm{m}$ (SD) along pathway (measured from the midline of the neural tube), and FL-CXCR4+ NCCs found $173 \pm 21.1 \mu \mathrm{m}$ (SD $p \leq 0.001)$. AtE4, control-EGFP + NCCs found $254 \pm 40.8 \mu \mathrm{m}$ (SD) and FL-CXCR4 + NCCs found $374 \pm 60.9 \mu \mathrm{m}$ (SD; $p \leq 0.001)$ along the dorsolateral pathway. $\boldsymbol{N}$, Schematic of measurements of cells along the dorsolateral pathway, graphed in $\boldsymbol{M}$. $\mathbf{0}-\boldsymbol{R}$ Images of control EGFP and FL-CXCR4 electroporated cells along the dorsolateral pathway with the migratory route shown in red. se, Surface ectoderm; nt, neural tube; no, notochord. Scale bars, $50 \mu \mathrm{m}$.

(see Fig. $8 A$ ). Thus, CXCR4 signaling functions to segregate NCCs between the DRGs and SGs.

\section{CXCR4-shRNA+ NCCs migrate more slowly than control cells}

We used time-lapse confocal microscopy to assess behaviors of CXCR4-shRNA + cells in whole trunk explants (HH St. 15) in which the majority of NCCs were colabeled with DiI. Time-lapse analysis $(n=5)$ showed that most CXCR4-shRNA+ cells stopped midway through the rostral somite (approximately the ventral edge of the presumptive DRGs), whereas nontransfected-DiI+ cells migrated further ventrally (Fig. 5A-E). DiI + NCCs that initially trailed CXCR4-shRNA+ cells, migrated around and past CXCR4shRNA+ cells distally along the migratory route. Quantification

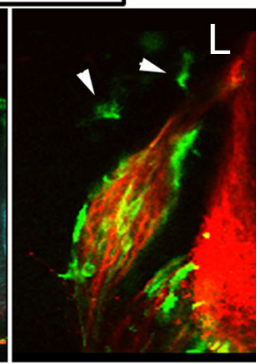

of cell velocity indicates that CXCR4shRNA + cells migrated slower $(9.8 \pm 1.5$ $\mu \mathrm{m} / \mathrm{h} ; n=5$ time-lapse sessions, 42 cells total) than control-DiI+ NCCs $(19.3 \pm 7.8$ $\mu \mathrm{m} / \mathrm{h} ; p=0.001 ; n=4$ time-lapse sessions, 45 cells total) (Fig. $5 F$ ). These velocities were calculated only during active migration and measurements ceased once the cells had stopped midway through the rostral somite (at the ventral level of the DRGs).

\section{What happens to CXCR4-shRNA+ NCCs?}

Although very few CXCR4-shRNA+ NCCs reached the presumptive SG location, those cells that did were primarily found along the (non-neural) perimeter of the ganglion, in contrast to the uniform distribution of transfected cells in control embryo SGs (Fig. 5G). At HH St. 24, $79.2 \pm 26 \%$ of CXCR4-shRNA NCCs ( $n=7$ embryos, 591 cells) were found along the SG perimeter compared with only $35.3 \pm 7.7 \%$ of control-shRNA transfected NCCs ( $n=4$ embryos, 201 cells), a 78\% increase above control (Fig. $5 G)(p=0.001)$. Thus, NCCs that reached the SGs and typically would have localized to the neural core of the ganglion, instead colonized the perimeter when CXCR4 signaling was disrupted.

Within the DRGs (HH St. 21), there was no difference in the percentage of EGFP+ neurons that differentiated into neurons between control-shRNAtransfected NCCs versus CXCR4-shRNAtransfected NCCs $(15.3 \pm 6.7$ vs $10.3 \pm$ $1.1 \%$ in controls; $p=0.1 ; n=4$ embryos each, 201 and 183 total cells counted, respectively). However, more CXCR4shRNA+ NCCs were localized to the dorsal root and DREZ than in controlshRNA transfected DRGs (Fig. 6A-C). We quantified the number of EGFP+ cells residing in the dorsal root region (dorsal one-fourth of the total DRGs measured dorsal to ventral) compared with the entire DRGs (length from the DREZ to motor exit point). In CXCR4-shRNAtransfected embryos, $24.2 \pm 5.9 \%$ of transfected NCCs $(n=4$ embryos, 183 cells) were found in the dorsal root compared with control embryos in which $11.4 \pm 4.4 \%$ of transfected cells $(n=4$ embryos, 201 cells) were in the dorsal root ( $p \leq 0.001)$; in contrast, the distribution of transfected cells within the DRG core was comparable (Fig. 6D,E) between conditions.

To confirm that the CXCR4-shRNA effects were target specific, we generated a second shRNA targeted to a different region of CXCR4 ( $n=6$ embryos). Transfected NCCs with both shRNAs show similar results with very few cells found in the SGs (with these restricted to the SG perimeter) and a higher percentage of transfected cells localizing to the dorsal pole of the DRGs (supplemental Fig. $2 B, C$, available at www.jneurosci.org as supplemental material). 
Additionally, to further support our findings that DRG formation is unaffected by CXCR4-SDF-1 signaling, we transfected CXCR4-shRNA into later stage embryos (HH St. 13 and 16), to specifically target those neural crest cells that primarily colonize the DRGs (George et al., 2010), which migrate after the bulk of the SG-destined NCCs. When DRG-destined NCCs are targeted for CXCR4 knock-down, no difference in cell distribution within the DRGs is obtained (supplemental Fig. 3, available at www.jneurosci.org as supplemental material).

\section{CXCR4 gain of function redirects NCC migration}

These data demonstrate that reductions in CXCR4 selectively abrogated the ventral migration of SG-destined NCCs. Based on this finding, we would predict that ectopic expression of CXCR4 might recruit additional NCCs to the ventral location where SGs form. To test this, we transfected premigratory NCC with a FL-CXCR4 expression plasmid that also encoded EGFP to track their migration and position within the embryo. We determined the locations of all FL-CXCR4+ NCCs using the same methods as in the previous experiments (Figs. 7, 8A,D). The distribution of transfected cells was opposite to that obtained when CXCR4 levels were reduced: transfection with CXCR4 induced a significant decrease in NCC colonization of the ipsilateral DRGs and an increase in the ipsilateral SGs. Of the FLCXCR4+ transfected cells that incorporated into the SGs, $82 \%$ colonized the neural core, compared with only $62 \%$ in controls (Fig. 7A-D). Furthermore, SGs in FL-CXCR4-transfected embryos were more compact compared with controls, which were less tightly aggregated. There was also a significant increase in FL-CXCR4 + cells along the dorsolateral pathway $(14.9 \pm 3.1 \mathrm{vs}$ $4.8 \pm 1.6 \%$ in controls; $p \leq 0.001$ ). At HH St. 18 (E3), FL-CXCR4 cells were found $173 \pm 21.1 \mu \mathrm{m}$ along the dorsolateral pathway, compared with $58.5 \pm 11.1 \mu \mathrm{m}$ in controls $(p=0.001)$ (Fig. 7O,Q). Additionally, we found $5.0 \pm 3.4 \%$ of FLCXCR $4+$ cells (compared with $0.2 \pm 0.5 \%$ in controls; $p \leq$ 0.003 ) in ectopic locations (where NCCs never reside) (e.g., lateral to the DRGs, perinotochordal, and in the contralateral SGs).

Interestingly, FL-CXCR4 + NCCs migrated widely not only on the ipsilateral but also on the contralateral side of the embryo (nonelectroporated side) (Fig. 7E-L). Previous studies from one of our laboratories (George et al., 2007) identified a subpopulation of trunk NCCs that cross the dorsal midline and colonize the contralateral DRGs but not other NCC-derived structures such as the SGs. Within the DRGs, the majority of contralateral cells were shown to colonize the non-neuronal regions of the DRGs including the dorsal pole and perimeter (George et al., 2007). Here, we report that FL-CXCR4+ NCCs were found not only throughout the contralateral DRGs, including the neural core at $\mathrm{HH}$ St. 24 (Fig. $7 I-L$ ), but also in the contralateral SGs $(8.4 \pm 3.9$ vs $0 \%$ in controls; $p \leq 0.001)$ with $91.8 \pm 8.25 \%$ in the neural core (Fig. $7 E-H$ ).

\section{Discussion}

A fundamental question in neural development has been to understand how a common pool of migrating NCCs sort themselves to stop at different locations along a shared migratory pathway to give rise to distinct structures of the PNS. The data reported here indicate that SG-destined NCCs are selectively targeted to their site of ganglion formation because of their differential expression of CXCR4 that confers the ability to respond chemotactically to an asymmetrically distributed ligand. We show with gain- and loss-of-function manipulations, that expression of CXCR4 is required to attract NCCs to the incipient SGs.

Chemotaxis has been hypothesized to be a mechanism for guiding NCCs to targets, particularly for cells that travel long distances (McGrath et al., 1999). Arguments against NCCs using chemotaxis as a guidance mechanism include the fact that NCCs typically migrate before their target tissue has fully developed, and that a cohort of NCCs can have multiple distinct targets along a shared, common migratory route. However, the data presented here are the first to demonstrate an in vivo role for chemotaxis in patterning the migration of trunk NCCs. In support of chemotaxis as a neural crest guidance mechanism, we found that only a subset of NCCs express CXCR4, and CXCR4+ cells are found in the SG anlagen but not in the DRG anlagen. Furthermore, the gain-of-function data reported here indicate that this selective, restricted expression of CXCR4 is crucial to ensure the normal distribution of NCCs to their various requisite targets. The temporal pattern of CXCR4 expression in neural crest is consistent with its mediating a role in SG-destined, but not DRG-destined NCCs, the majority of which colonize the DRGs after CXCR4 expression ceases in the region where the DRG anlagen will form (Serbedzija et al., 1989; George et al., 2007). It will be of interest to identify factors regulating the tight temporal and spatial expression of CXCR4 in only a distinct subset of NCCs.

These data demonstrate that a subset of SG-destined NCCs are molecularly distinct from their larger cohort. We suggest that differ- 
ential expression of CXCR4 generates two distinct subpopulations of neural crest cells that initially share a common pathway, yet subsequently diverge because of their differential response to SDF-1. This is analogous to the selection of target choices for sympathetic neurons in the superior cervical ganglion: the endothelin receptor Endra is expressed on the subset of sympathetic neurons that selectively extend along the endothelin-3-secreting external carotid artery (Makita et al., 2008). Thus, both studies demonstrate that differential expression of a particular guidance receptor prepatterns the target selection of sympathetic precursors in the neural crest, and of neurons in sympathetic ganglia.

In light of evidence that SDF-1 reduces the effectiveness of multiple axon repellents, including slits and semaphorins (Chalasani et al., 2003), we hypothesize that CXCR4-SDF-1 signaling enables SG-destined NCCs to migrate through potentially inhibitory environments to arrive at the dorsal aorta. As they migrate to the dorsal aorta, NCCs must traverse the perinotochordal area, a region rich in inhibitory molecules (Tosney, 1991). Slit-1, -2, and -3 are expressed in the ventral neural tube, notochord, and near the dorsal aorta (De Bellard et al., 2003), all regions in close proximity to the migratory pathway along which NCCs travel to arrive at the SGs. Since NCCs in the DRGs fail to express CXCR4, they would be unable to penetrate into this inhibitory region and hence would stop more dorsally and form the DRGs. Thus, differential expression of CXCR4 might determine whether NCCs travel long or short distances along a common migratory route and hence pattern the colonization of NCCs to specific "stop" sites. These data are additionally supported by the fact that NCCs ectopically expressing CXCR4 were detected in locations that are typically inhibitory to NCCs: the perinotochordal region and along the dorsolateral pathway, which initially repels NCCs because of eph/ephrin interactions (Santiago and Erickson, 2002). Furthermore, ectopic expression of CXCR4 caused more NCCs to migrate contralaterally than is normal, again, perhaps failing to respond to an inhibitory signal that would normally restrict them to the ipsilateral pathway (George et al., 2007). These data suggest that a normal function for CXCR4 is not only to guide NCCs chemotactically toward the dorsal aorta, but it does so by abrogating signaling pathways that would otherwise restrict the directional decisions made by NCCs, especially within inhibitory regions.

In the absence of a chemotactic gradient, how NCCs would be directed through an inhibitory zone is not clear. If CXCR4/SDF-1 signaling is modulating inhibitory signals, NCCs could be directed by physical contact, such as contact inhibition of movement. Recent evidence in zebrafish has shown NCCs display contact inhibition of movement. In the presence of local inhibitory signals, contact inhibition has been suggested to direct NCC migratory streams (Carmona-Fontaine et al., 2008). However, our time-lapse analysis reveals trunk NCCs migrate as individuals or in chain-like arrays to the SGs, demonstrating that contact inhibition is insufficient to explain the complex behaviors exhibited by NCCs as they migrate through the trunk (Kasemeier-Kulesa et al., 2005).

A second possible model that could account for the aberrant navigation and decreased migratory speed of CXCR4-shRNA+ sympathetic precursors is that CXCR4 signaling modulates cell contact with the extracellular matrix (ECM). For example, CXCR4 is highly expressed in several neuroblastoma cell lines and overexpression leads to increased metastasis (Zhang et al., 2007). SDF-1 can upregulate integrins through CXCR4 in migrating chondrosarcoma and lung cancer cells (Huang et al., 2007; Lai et al., 2009) and integrins are crucial for avian trunk NCC migration on fibronectin (Kil et al., 1998). Decreasing CXCR4 signaling in NCCs may decrease integrin expression and thereby reduce the ability of cells to efficiently attach to the ECM and limit their migratory speed and ability to travel deep within the embryo. As a result, NCCs would pool more dorsally (Figs. 6, 8). In contrast, ectopic overexpression of CXCR4 could enhance NCC interactions with the ECM thereby indiscriminately promoting motility and migration, at the expense of their normal appropriate response to the non-ECM, stop signals and cell adhesion cues (Figs. 7, 8). Our shRNA data are also consistent with a role for CXCR4 signaling in regulating cadherin expression since ectopic expression of CXCR4 increased the compaction of SG. It has been shown that the protocadherin pcdh-1 plays a key role in positioning cells within either the neural core versus perimeter of chick DRGs (Bononi et al., 2008).

Many studies have identified developmental roles for SDF-1/ CXCR4 signaling (Bagri et al., 2002; Jaleel et al., 2004; Vasyutina et al., 2005). In the zebrafish, lateral line primordium cells are guided to their target location by chemokine signaling and blocking CXCR4 on lead lateral line cells prevents proper navigation of the entire cluster (Valentin et al., 2007). Similar to chick, a subset of mouse NCCs express CXCR4 and SDF-1 is expressed along their migratory route (Belmadani et al., 2005). This study also showed that, in vitro, mouse NCCs could chemotax toward SDF-1. Although the identity of the CXCR4+ subpopulation of NCCs was not determined in the mouse nor was SG development examined in CXCR4-null mice, the genetic deletion of CXCR4 altered DRG morphology (Belmadani et al., 2005). Thus, both studies demonstrate an important function for CXCR4/SDF1 signaling in PNS development. Recently, it has been shown that CXCR4 plays yet a different role in zebrafish cranial NCC migration; although zebrafish trunk NCCs do not express CXCR4, reductions in CXCR4 interfered with cranial ganglia development (Olesnicky Killian et al., 2009). In summary, CXCR4, plays a key role in NCC migration in all three species, although it apparently does not regulate the migration of the same subpopulation of NCCs.

Our data indicate that the ventrally migrating pool of NCCs is molecularly heterogeneous. This molecular heterogeneity could be the manifestation of temporal differences in delamination in that the CXCR4+ NCC cohort delaminates in the first wave of emigration from the neural tube. Whatever cues might induce the early wave of NCCs to express CXCR4 may no longer be present once the subsequent waves of neural crest delaminate. Although transplantation studies clearly indicate a tremendous degree of plasticity among NCCs, differential expression of CXCR4 is not necessarily an indication of cell commitment, but rather cell specification. According to this model, expression of CXCR4 facilitates ventral migration to the dorsal aorta, where the CXCR4+ cells will receive cues such as BMP4 that will induce MASH (mammalian achaete/scute homolog) expression, and commit these cells to a sympathetic neural fate.

In summary, our data indicate chemokine signaling may be crucial for proper trunk NCC guidance and potentially for the distribution or balance of NCCs that target DRGs or SGs. For example, at a particular somite level, in response to embryological manipulations that cause a significant increase in the number of NCCs that contribute to the DRGs, there is a corresponding decrease in cells in the SGs (Goldstein and Kalcheim, 1991). This could partially be explained by intercellular signaling among the pool of NCCs that will colonize DRGs and SGs within a particular axial level. Evidence for such cross talk was observed in our timelapse sequences in which NCCs were observed at early ages moving back and forth between presumptive SG and DRG sites at a given axial level (Kasemeier-Kulesa et al., 2005). Additional insights into the interplay of molecular signals that regulate trunk NCC invasion 
and distribution into specific structures may shed insight on mechanisms underlying hereditary sensory and autonomic neuropathies and the neural crest-derived cancer, neuroblastoma.

\section{References}

Bagri A, Gurney T, He X, Zou YR, Littman DR, Tessier-Lavigne M, Pleasure SJ (2002) The chemokine SDF1 regulates migration of dentate granule cells. Development 129:4249-4260.

Belmadani A, Tran PB, Ren D, Assimacopoulos S, Grove EA, Miller RJ (2005) The chemokine stromal cell-derived factor-1 regulates the migration of sensory neuron progenitors. J Neurosci 25:3995-4003.

Bononi J, Cole A, Tewson P, Schumacher A, Bradley R (2008) Chicken protocadherin-1 functions to localize neural crest cells to the dorsal root ganglia during PNS formation. Mech Dev 125:1033-1047.

Britsch S, Li L, Kirchhoff S, Theuring F, Brinkmann V, Birchmeier C, Riethmacher D (1998) The ErbB2 and ErbB3 receptor and their ligand, neuregulin-1, are essential for development of the sympathetic nervous system. Genes Dev 12:1825-1836.

Carmona-Fontaine C, Matthews HK, Kuriyama S, Moreno M, Dunn GA, Parsons M, Stern CD, Mayor R (2008) Contact inhibition of locomotion in vivo controls neural crest directional migration. Nature 456:957-961.

Chalasani SH, Sabelko KA, Sunshine MJ, Littman DR, Raper JA (2003) A chemokine, SDF-1, reduces the effectiveness of multiple axonal repellents and is required for normal axon pathfinding. J Neurosci 23:1360-1371.

De Bellard ME, Rao Y, Bronner-Fraser M (2003) Dual function of Slit2 in repulsion and enhanced migration of trunk, but not vagal, neural crest cells. J Cell Biol 162:269-279.

Elbashir SM, Martinez J, Patkaniowska A, Lendeckel W, Tuschl T (2001) Functional anatomy of siRNAs for mediating efficient RNAi in Drosophila melanogaster embryo lysate. EMBO 20:6877-6888.

George L, Chaverra M, Todd V, Lansford R, Lefcort F (2007) Nociceptive sensory neurons derive from contralaterally migrating, fate-restricted neural crest cells. Nat Neurosci 10:1287-1293.

George L, Kasemeier-Kulesa J, Nelson BR, Koyano-Nakagawa N, Lefcort F (2010) Patterned assembly and neurogenesis in the chick dorsal root ganglion. J Comp Neurol 518:405-422.

Goldstein RS, Kalcheim C (1991) Normal segmentation and size of the primary SG depend upon the alternation of rostrocaudal properties of the somites. Development 112:327-334.

Hamburger V, Hamilton HL (1951) A series of normal stages in the development of the chick embryo. J Morphol 88:49-92.

Howard MJ, Stanke M, Schneider C, Wu X, Rohrer H (2000) The transcription factor dHAND is a downstream effector of BMPs in sympathetic neuron specification. Dev Biol 127:4073-4081.

Huang YC, Hsiao YC, Chen YJ, Wei YY, Lai TH, Tang CH (2007) Stromal cell-derived factor-1 enhances motility and integrin up-regulation through CXCR4, ERK and NF- $\kappa \mathrm{B}-$ dependent pathway in human lung cancer cells. Biochem Pharmacol 74:1702-1712.

Jaleel MA, Tsai AC, Sarkar S, Freedman PV, Rubin LP (2004) Stromal cellderived factor-1 (SDF-1) signaling regulates human placental trophoblast cell survival. Mol Hum Reprod 10:901-909.

Jiang Y, Liu MT, Gershon MD (2003) Netrins and DCC in the guidance of migrating neural crest-derived cells in the developing bowel and pancreas. Dev Biol 258:364-384.

Kalatskaya I, Berchiche YA, Gravel S, Limberg BJ, Rosenbaum JS, Heveker N (2009) AMD3100 is a CXCR7 ligand with allosteric agonist properties. Mol Pharmacol 75:1240-1247.

Kasemeier JC, Lefcort F, Fraser SE, Kulesa PM (2004) A novel sagittal slice explant technique for time-lapse imaging of the formation of the chick peripheral nervous system. In: Imaging in neuroscience and development (Yuste R, Konnerth A, eds). Cold Spring Harbor, NY: Cold Spring Harbor Laboratory.

Kasemeier-Kulesa JC, Kulesa PM, Lefcort F (2005) Imaging neural crest cell dynamics during formation of dorsal root ganglia and sympathetic ganglia. Development 132:235-245.

Kasemeier-Kulesa JC, Bradley R, Pasquale EB, Lefcort F, Kulesa PM (2006) Eph/ephrins and N-cadherin coordinate to control the pattern of sympathetic ganglia. Development 133:4839-4847.

Kawasaki T, Bekku Y, Suto F, Kitsukawa T, Taniguchi M, Nagatsu I, Nagatsu T, Itoh K, Yagi T, Fujisawa H (2002) Requirement of neuropilin 1 -mediated Sema3A signals in patterning of the sympathetic nervous system. Development 129:671-680.
Kil SH, Krull CE, Cann G, Clegg D, Bronner-Fraser M (1998) The alpha4 subunit of integrin is important for neural crest cell migration. Dev Biol 202:29-42.

Kulesa PM, Kasemeier-Kulesa JC (2007) Construction of a heated incubation chamber around a microscope stage for time-lapse imaging. Cold Spring Harb Protoc 2007:4792.

Kulesa PM, Teddy JM, Stark DA, Smith SE, McLennan R (2008) Neural crest invasion is a spatially-ordered progression into the head with higher cell proliferation at the migratory front as revealed by the photoactivatable protein, KikGR. Dev Biol 316:275-287.

Kulesa PM, Lefcort F, Kasemeier-Kulesa JC (2009) The migration of autonomic precursor cells in the embryo. Auton Neurosci 151:3-9.

Kuriyama S, Mayor R (2008) Molecular analysis of enural crest migration. Philos Trans R Soc Lond B Biol Sci 363:1349-1362.

Lai TH, Fong YC, Fu WM, Yang RS, Tang CH (2009) Stromal cell-derived factor-1 increase alphavbeta3 integrin expression and invasion in human chondrosarcoma cells. J Cell Physiol 218:334-342.

LeDouarin NM, Kalcheim C (1999) The neural crest. Cambridge, UK: Cambridge UP.

Makita T, Sucov HM, Gariepy CE, Yanagisawa M, Ginty DD (2008) Endothelins are vascular-derived axonal guidance cues for developing sympathetic neurons. Nature 452:759-763.

McGrath KE, Koniski AD, Maltby KM, McGann JK, Palis J (1999) Embryonic expression and function of the chemokine SDF-1 and its receptor, CXCR4. Dev Biol 213:442-456.

Miller RJ, Banisadr G, Bhattacharyya BJ (2008) CXCR4 signlaing in the regulation of stem cell migriaton and development. J Neuroimmunol 198:31-38.

Olesnicky Killian EC, Birkholz DA, Artinger KB (2009) A role for chemokine signaling in neural crest cell migration and craniofacial development. Dev Biol 333:161-172.

Raz E, Mahabaleshwar H (2009) Chemokine signaling in embryonic cell migration: a fisheye view. Development 136:1223-1229.

Reichenbach B, Delalande JM, Kolmogorova E, Prier A, Nguyen T, Smith CM, Holzschuh J, Shepherd IT (2008) Endoderm-derived Sonic hedgehog and mesoderm Hand2 expression are required for enteric nervous system development in zebrafish. Dev Biol 318:52-64.

Reissmann E, Ernsberger U, Francis-West PH, Rueger D, Brickell PM, Rohrer $H$ (1996) Involvement of bone morphogenetic protein-4 and bone morphogenetic protein-7 in the differentiation of the adrengergic phenotype in developing sympathetic neurons. Development 122:2079-2088.

Santiago A, Erickson CA (2002) Ephrin-B ligands play a dual role in the control of neural crest cell migration. Development 129:3621-3632.

Serbedzija GN, Bronner-Fraser M, Fraser SE (1989) A vital dye analysis of the timing and pathways of avian trunk neural crest cell migration. Development 106:809-816.

Stark DA, Kulesa PM (2007) An in vivo comparison of photoactivatable fluorescent proteins in an avian embryo model. Dev Dyn 236:1583-1594.

Tosney KW (1991) Cells and cell-interactions that guide motor axons in the developing chick embryo. Bioessays 13:17-23.

Tran PB, Ren D, Veldhouse TJ, Miller RJ (2004) Chemokine receptors are expressed widely by embryonic and adult neural progenitor cells. J Neurosci Res 76:20-34.

Tsarovina K, Schellenberger J, Schneider C, Rohrer H (2008) Progenitor cell maintenance and neurogenesis in sympathetic ganglia involves Notch signaling. Mol Cell Neurosci 37:20-31.

Valentin G, Haas P, Gilmour D (2007) The chemokine SDF1a coordinates tissue migration through the spatially restricted activation of Cxcr7 and Cxcr4b. Curr Biol 17:1026-1031.

Vasyutina E, Stebler J, Brand-Saberi B, Schulz S, Raz E, Birchmeier C (2005) CXCR4 and Gab1 cooperate to control the development of migrating muscle progenitor cells. Genes Dev 19:2187-2198.

Young HM, Hearn CJ, Farlie PG, Canty AJ, Thomas PQ, Newgreen DF (2001) GDNF is a chemoattractant for enteric neural cells. Dev Biol 229:503-516.

Young HM, Anderson RB, Anderson CR (2004) Guidance cues involved in the development of the peripheral autonomic nervous system. Auton Neurosci 112:1-14.

YusufF, Rehimi R, Dai F, Brand-Saberi B (2005) Expression of chemokine receptor CXCR4 during chick embryo development. Anat Embryol 210:35-41.

Zhang L, Yeger H, Das B, Irwin MS, Baruchel S (2007) Tissue microenvironment modulates CXCR4 expression and tumor metastasis in neuroblastoma. Neoplasia 9:36-46. 